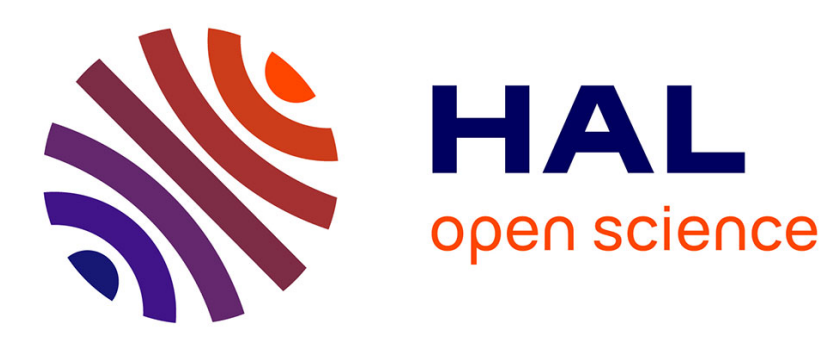

\title{
Darrieus-Landau instability of premixed flames enhanced by fuel droplets
}

\author{
Colette Nicoli, Pierre Haldenwang, Bruno Denet
}

\section{To cite this version:}

Colette Nicoli, Pierre Haldenwang, Bruno Denet. Darrieus-Landau instability of premixed flames enhanced by fuel droplets. Combustion Theory and Modelling, 2017, 21 (4), pp.630 - 645 . 10.1080/13647830.2017.1279756 . hal-01678255

\section{HAL Id: hal-01678255 \\ https://hal.science/hal-01678255}

Submitted on 7 May 2018

HAL is a multi-disciplinary open access archive for the deposit and dissemination of scientific research documents, whether they are published or not. The documents may come from teaching and research institutions in France or abroad, or from public or private research centers.
L'archive ouverte pluridisciplinaire HAL, est destinée au dépôt et à la diffusion de documents scientifiques de niveau recherche, publiés ou non, émanant des établissements d'enseignement et de recherche français ou étrangers, des laboratoires publics ou privés. 


\title{
Darrieus-Landau instability of premixed flames enhanced by fuel droplets
}

\author{
Colette Nicoli, Pierre Haldenwang*, \\ Aix Marseille Université, CNRS, Centrale Marseille, \\ M2P2 UMR 7340, 13451, Marseille, France \\ and \\ Bruno Denet \\ Aix Marseille Université, CNRS, Centrale Marseille, \\ IRPHE UMR 7342, 13384, Marseille, France
}

December 6, 2016

\begin{abstract}
Recent experiments on spray-flames propagating in Wilson cloud chamber have established that spray-flames are much more sensitive to wrinkles or corrugations than single-phase flames. To propose certain elements of explanation, we numerically study the Darrieus-Landau (or hydrodynamic) instability developing in premixtures that contain an array of fuel droplets. Two approaches are compared: the numerical simulation starting from the general conservation laws in reactive media (DNS), and the numerical computation of Sivashinsky-type modelequations (SME) for DL-instability. Both approaches provide us with results in deep agreement.

It is first shown that the presence of droplets in fuel/air premixtures induces initial perturbations which are large enough to trigger the Darrieus-Landau (DL) instability. Second, the droplets are responsible of additional wrinkles when the DL instability is developed. The latter wrinkles are of length scales shorter than those of the DL-instability, in such a way that the DL-unstable spray-flames have a larger front surface and therefore propagate faster than the single-phase ones when subjected to the same instability.
\end{abstract}

\footnotetext{
${ }^{*}$ Corresponding author

${ }^{0}$ Preprint submitted to Combustion Theory and Modelling
} 
keyword:

spray-flame; two-phase combustion; Darrieus-Landau instability; hydrodynamic instability; droplet combustion

\section{Nomenclature}

30 $\delta_{f}^{*} \quad$ characteristic thickness of the stoichiometric single-phase flame

$\gamma \quad$ expansion parameter

$\psi \quad$ front position in Sivashinsky DL-model equation

$\rho_{G} \quad$ density of the fuel vapour

$\rho_{L} \quad$ density of the fuel liquid phase

$35 \tau_{f}^{*} \quad$ characteristic transit time of the stoichiometric single-phase flame

$\tau_{\text {prop }} \quad$ characteristic time of flame propagation between two droplets

$\tau_{v a p} \quad$ characteristic time of droplet vaporization according to D-2 law

$\varphi_{G} \quad$ equivalence ratio of the fuel-saturated premixture

$\varphi_{L} \quad$ equivalence ratio of fuel under liquid phase to overall quantity of air

$40 \quad \varphi_{T} \quad$ overall equivalence ratio of the spray

$\varphi_{u} \quad$ local equivalence ratio of unburnt gases upstream of the non-homogeneous flame

$D_{t h, b}^{*}$ thermal diffusivity of burnt gases for a stoichiometric mixture

$L_{d} \quad$ typical droplet interdistance

$45 \quad L_{x} \quad$ length of the computational domain, parallel to flame propagation

$L_{y} \quad$ height of the computational domain, transverse to flame propagation

$L_{D L} \quad$ computational box height above which DL-instability develops

$\mathrm{Pe}_{s} \quad$ spray Péclet number

$R_{d} \quad$ droplet radius

${ }_{50} \quad s \quad$ lattice spacing $\left(s=L_{d} \sqrt{2}\right)$ 
$T_{b}^{*} \quad$ adiabatic temperature of the stoichiometric single-phase flame

$U_{L} \quad$ single-phase laminar flame velocity

$U_{L}^{*} \quad$ adiabatic single-phase laminar flame velocity at stoichiometry

$x_{F} \quad$ mean front position

55

\section{Introduction}

In combustion systems, fuel is often injected under liquid phase as in diesel, aerospace engines or furnaces. This has long motivated studies dealing with spray-flame dynamics. The experimental literature reports numerous situations where the spray-flame is more corrugated, and faster, than the equivalent single-phase flame. We have particularly in mind the recent experiments conducted in Wilson chamber [1] and [2, the latter experiments being performed in microgravity. Consequent wrinkles have also been reported in earlier works about spray-flames [3, 4, 5], or particle cloud combustion [6]. There is hence an important number of observations which indicate that spray-flame fronts are particularly prone to fold, if the droplet size is large enough. The purpose of the present numerical work is to contribute to understand this phenomenon.

The experiments on combustion in sprays at moderate and high pressures have generally revealed behaviors in large departure from the equivalent single-phase premixed flames. For instance, two-phase flames have been found to propagate with a pulsating regime [7, 6] without the resort to differential diffusive effects [8, 9, 10]. In addition, certain results obtained in microgravity [11] have shown that spray-flames in lean ethanol-air mixtures can propagate faster than the equivalent gaseous flames, and slower in globally rich mixtures. Recent numerical studies [12, 13] concerning globally lean mixtures with a fixed overall equivalence ratio $\varphi_{T}=0.85$ brings complementary results concerning the mixture composition effects and the droplet size influence on spray-flame dynamics. On the other hand, classical ground experiments [3, 4, 1] have exhibited opposite observations to the ones made in [11]: spray-flames in rich mixtures of ethanol (or iso-octane) and air are faster than the equivalent gaseous premixed flame.

To interpret these complex aspects of the flame propagation in dropletvapor-air mixture, Hayashi and Kumagai [3, 4] introduced the concept of effective fuel-air ratio. This corresponds to an attempt at accounting for the unvaporized fuel and incompletely mixed fuel, which depends in a complex 
manner on droplet size and fuel-air ratio. Our recent investigations on sprayflames dynamics [12, 14, 15] have established the various roles played by the main spray parameters: $\varphi_{G}$, the equivalence ratio of the fuel-saturated premixture that initially surrounds the droplets, $\varphi_{L}$, the liquid loading or the equivalence ratio of the fuel under liquid phase to the overall quantity of air, and $R_{d}$, the typical droplet radius. Note that we obviously have $\varphi_{T}=\varphi_{G}+\varphi_{L}$ and $L_{d}$, the typical droplet interdistance, is a function of $R_{d}$ and $\varphi_{L}$. In particular, in both recent contributions [14, 15] on rich sprayflames, we have proposed to consider the "spray Péclet number" defined as follows:

$$
P e_{s} \propto \frac{\rho_{L}}{\rho_{G}} \frac{2 R_{d} * U_{L}\left(\varphi_{G}\right)}{D_{t h, b}^{*}} \frac{2 R_{d}}{L_{d}}
$$

The "spray Péclet number" corresponds to the ratio of the droplet vaporization time $\tau_{\text {vap }} \propto\left(\rho_{L} / \rho_{G}\right)\left(2 R_{d}\right)^{2} / D_{\text {th }, b}^{*}$ to the propagation time $\tau_{\text {prop }} \propto$ $L_{d} / U_{L}\left(\varphi_{G}\right)$, where $U_{L}\left(\varphi_{G}\right)$ and $D_{t h, b}^{*}$ are the laminar single-phase flame speed related to the initial fuel-saturated mixture and the thermal diffusivity in the burnt gases of the stoichiometric single-phase laminar flame, respectively. Note that $R_{d}$, the $2 \mathrm{D}$ droplet radius (or more properly the disk radius), is a function of both droplet interdistance $L_{d}$ and liquid loading $\varphi_{L}$. This obviously takes account of the present 2D planar geometry, so that the actual $2 \mathrm{D}$ radius is about one third of the radius that would give the same liquid equivalence ratio in $3 \mathrm{D}$.

When the "spray Péclet number" is large, the flame propagation between two droplets is so fast that droplet vaporization does not modify the initial premixture that surrounds the droplets. In other words, spray-flame 95 speed is simply the one of the single-phase flame propagating in the initial premixture. This result has numerically been established clearly, as long as the spray-flame remained planar. Now, to simplify the forthcoming discussions on folded flames, we decide to assume that the "spray Péclet number" is large, in such a way that the role of the droplets is restricted to create small regions of rich combustion imposed to a single-phase flame front locally propagating with $U_{L}\left(\varphi_{G}\right)$. Accordingly with the concept of effective fuel-air ratio introduced by Hayashi and Kumagai [3, 4, the effective fuel-air ratio is here reduced to $\varphi_{G}$ for spray with $P e_{s}>\mathcal{O}(1)$.

A situation where the "spray Péclet number" is large generally corresponds to a rich spray. Even though engines are desirable in lean configuration, rich spray-flames are known to locally exist close to the system of liquid injection.

Because in numerous experiments the spray-flame front was found cor- 
rugated with a large number of cells, it is a difficult task to determine the fundamental laminar velocity of the spray-flames from those experimental data. Moreover, the spray-flame speed enhancement observed in the experiments could possibly be explained by instabilities of the front, only. As a matter of fact, the complex interplay between instabilities and droplets seems to have a peculiar importance for the spherical flames, a case that has extensively been studied in the recent years [11, 16, 1], and that follows the pioneering work of Hayashi and Kumagai (1975) 3]. The present work, which adopts the simplified configuration $P e_{s}>\mathcal{O}(1)$, allows us to cancel the part of the interplay in relation with the role of the vaporization in the premixture establishment. On the theoretical side, the creation of many cells in spherical premixed flames (without droplets) has been modeled with the Sivashinsky equation, particularly in 2D by Karlin and Sivashinsky (2007) [17]. This DL-model equation contains the main two effects, creation of many cells and self-acceleration.

\section{Two models for DL-unstable spray-flame prop- agation}

The article is based on the predictions of two different approaches that are able to study the Darrieus-Landau instability (or the hydrodynamic instability of premixed flame). The first one uses the 2D numerical simulation (DNS) that starts from the basic equations characterizing any reactive medium. As for the second approach, we use a much lighter mathematical approach (SME) that corresponds to the DL-model equation due to Sivashinsky.

\subsection{Solving the conservation 2-D laws for reactive media (DNS)}

135 This model of spray-flame propagation resorts to the general approach that we have used in our recent contributions [12, 14]. The initial state of the spray is schematized by a $2 \mathrm{D}$ face-centered lattice, at the nodes of which the droplets are positioned. The lattice spacing is denoted by $s$, in such a way that the droplet interdistance is $L_{d}=s / \sqrt{2}$. The spray-flame propagation is governed by the usual set of conservation laws for mass, momenta, energy and species, with the simplest chemical scheme [14] that allows us to handle a flame propagating through a medium of heterogeneous composition, where the equivalence ratio varies from $\varphi_{G}$ far from the droplets to a very large 


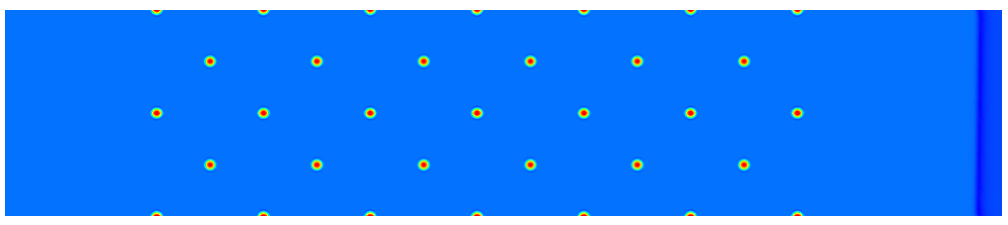

Figure 1: Alkane/air droplet lattice: initial fuel mass fraction resulting from the positioning of the droplets at the nodes of a 2D-lattice of spacing $s$. On the very right side of the figure, a planar single-phase premixed flame propagates to the left (in the lattice: $\varphi_{G}=1.1, \varphi_{L}=0.5, s=12$ ). The computational domain represented is $L_{y}=2 \times s$ hight and $L_{x}=9.7 \times s$ long.

value close to the droplets. As described in [14], the spray is considered as a non-homogeneous one-phase medium. Vaporization, Stefan flow and heatmass transfers at the liquid-gas interface are solved from the conservation laws and do not require particular sub-models.

By contrast with most literature on spray-flames (for a recent review, see [18]), the current droplets are here fully resolved. This explains why 3-D DNS is hardly affordable for a parametric exhaustive study. The droplet radius, being a function of both lattice spacing and liquid loading, has a typical value which varies in a range from 0.1 to 1 in flame thickness units. The droplets belong to the same continuum as the gas; therefore, they can move, heat, follow the properties of a real gas, and continuously switch from (liquid) dense fluid to (vapour) light fluid. The present simulation takes advantage of the fact that latent heat and surface tension are neglected for the following reasons: a) latent heat of alkanes corresponds to a small fraction of the reaction heat (e.g. see Ref.[19]) and can be neglected in the thermal budget of the pre-heat zone; b) liquid fuel containment can simply be achieved by the fact that heat and mass transfers are frozen at the spray initial temperature (the spray being at rest far upstream of the flame). The latter point is carried out by using the non-linear dependence of the diffusion coefficients with respect to temperature.

The droplets are initially set identical at the nodes of a face-centered lattice. The vaporization process is hence freed from the possible weakness related to the vaporization model selected. Note additionally that the experiments in cloud Wilson chambers, carried out in microgravity [2], lead to nearly monodisperse sprays, with a nearly regular density for the droplets. 
This is why the current initial conditions are seemingly relevant with regard to such experiments.

The chemical scheme, based on the classical one-step Arrhenius law, can be interpreted as resulting from a flamelet theory: the flame front is conceived as a series of small planar flames connected to each other, that individually propagate through a locally homogeneous medium. This consideration requires that the spray-flame thickness remains small in comparison with the typical droplet interdistance. This requirement is generally fulfilled for sprays at moderate and high pressures. The dynamics of these flames depends on the equivalence ratio of the local premixture burnt by each flamelet. More precisely, the local equivalence ratio, denoted by $\varphi_{u}$, is assessed [14] at each computational point in such a way that it represents the "upstream" equivalence ratio, as seen ahead by each small planar flame. Furthermore, it is known that the classical one-step Arrhenius law largely overestimates the adiabatic flame temperature of the rich mixture.

To overcome the difficulty to correctly assess the laminar flame speed, hereinafter denoted $U_{L}$, we considered an easy modification [14] of this simple scheme: heat release is a linear function of $\varphi_{u}$, the fresh premixture equivalence ratio. This model has been proven to correctly mimic the premixed single-phase flame behaviors (adiabatic flame temperature and flame speed) [20]. As a matter of fact, such a modification of heat release takes account of all the species existing at the actual flame temperature. From the numerical point of view, at each time step, the classical system of reactiondiffusion equations is solved first. Then, it is coupled with the Navier-Stokes equations. The overall scheme that computes the Navier-Stokes equations within the context of DL-instability has previously been described in Denet and Haldenwang [21].

The set of equations, as well as the results we shall present, are handled under non-dimensional form. The scales retained for non-dimensioning are those of the adiabatic, stoichiometric, premixed flame of the considered fuel (presently a heavy alkane). More precisely, the units for time, length, mass and temperature are derived from the theoretical properties of this ideal flame, in terms of flame thickness $\delta_{f}^{*}=D_{t h, b}^{*} / U_{L}^{*}$, transit time $\tau_{f}^{*}=D_{t h, b}^{*} / U_{L}^{* 2}$, flame temperature $T_{b}^{*}$ and burnt gas density $\rho_{b}^{*}$. Hereinafter, the subscript "b" is associated with the burnt mixture, at the flame temperature $T_{b}$. The superscript " *" is associated with the values assessed for the adiabatic, stoichiometric, single-phase flame. 
The numerical experiments are conducted as follows. The droplets are initially positioned at the nodes of a face-centred lattice of spacing $s$ (in $\delta_{f}^{*}$ units), as illustrated by Fig.1. The lattice is embedded in a computational box of length $L_{x}$ and height $L_{y}$. For periodicity reason, we have chosen either $L_{y}=s$ or $L_{y}=2 s$. The surface tension of the droplets being neglected, a droplet appear as a dense fuel puff belonging to the same continuum as its surrounding premixture. Furthermore, the containment of the fuel within the droplet results from the freezing of the mass diffusion at the unburnt temperature. The droplets can move, carried by the flow resulting from gas expansion. Heat-mass transfers, vaporization and the related gas expansion start when the medium is heated by the proximity of the flame.

If $s$ and $\varphi_{L}$ are given, $R_{d}$, the droplet radius is hence determined. In addition, if $\varphi_{G}$ is given, we can estimate the "spray Péclet number". All the numerical experiments considered in what follows satisfy the condition $P e_{s}>\mathcal{O}(1)$. Therefore, accordingly with our recent studies [14], [15, the presence of droplets has no impact on the general dynamics of the planar spray-flame. We shall show that this is no longer the case when the sprayflame is DL-unstable. Among the main results, the spray-flame speed is considered. For its evaluation, we have to define the rate of flame propagation. As the front is strongly corrugated and changing in space and time, we choose an averaged definition of the front position by performing the partial integration of the temperature field in the transverse (periodic) $y$-direction. We hence obtain the one-dimensional quantity $<T>_{y}(x)$. We then define $x_{F}$, the front position, as the locus where $<T>_{y}\left(x_{F}\right)=0.5$.

\subsection{Solving the Sivashinsky DL-model equation (SME)}

The results of our DNS that will be shown below suggest that if the premixture between droplets is not lean, the main effect of the droplets is to locally slow down the premixed flame. We suggest to model this phenomenon by using a variant of the Sivashinsky equation proposed by Joulin and Cambray for large gas expansion [22]. Note that, originally, the Sivashinsky DL-model was derived within the context of small gas expansion. The extension proposed by Joulin-Cambray is known to allow the description of larger gas expansion (the current gas expansion through the single-phase flames of our numerical experiments is about 5 , a value reputed affordable by the JoulinCambray extension). The idea is to include a position-dependent flame 
velocity in the non-linear term of this model-equation. It reads

$$
\begin{array}{r}
\psi_{t}+U_{e f f}(x, y)\left(1+\frac{a(\gamma)}{2} \psi_{x}^{2}\right)+\frac{(1-a(\gamma))}{2}<U_{e f f}(x, y) \psi_{x}^{2}> \\
=\Omega(\gamma) U_{g}\left(I(\psi)+\psi_{x x} / k_{n}\right)
\end{array}
$$

235

where $\psi$ is the position of the front $\gamma=\left(T_{b}-T_{u}\right) / T_{b}=0.8$ is the expansion parameter, $\Omega(\gamma)=\left((\gamma+1 /(1-\gamma))^{1 / 2}-1\right) /(2-\gamma)$ is the exact Darrieus Landau coefficient, $a(\gamma)$ is a coefficient controlling the flame amplitude given in [22]. $I(\psi)$ corresponds to the multiplication by $|k|$ in Fourier space, $k_{n}$ corresponding to the number of unstable modes in a box of width $2 \pi$. $U_{\text {eff } f}$ is an effective normal propagation speed, position-dependent, i.e. equal to $U_{g}=1$, the laminar flame propagation speed, outside the droplets, and to $U_{g} / 5$ in a circle of influence around each droplet. Note that this lowered flame velocity accounts for different effects: a very rich and slow flame around the droplets and an hydrodynamic effect of the flow field caused by droplet evaporation.

5 The Sivashinsky equation will be solved in various cases corresponding to a different number of unstable modes of the Darrieus-Landau instability (the stationary solutions of the equation without droplets being reported in [23] for different numbers of unstable modes).

The general idea sustaining the SME approach consists in checking whether s a Sivashinsky DL-model, that incorporates some local weak propagation, is able to retrieve the overall properties of the spray-flame. After several attempts for choosing both the size of the droplet influence zone and the drop in flame velocity, it turned out that varying both depth and size of the hole did not greatly change the results, provided that the size remains reasonably small in comparison with the lattice-spacing.

\section{Triggering DL-instability with droplets}

The classical theory of DL-instability indicates that the development of DLinstability within the framework of periodic conditions transverse to the propagation generally requires a wide enough "channel", namely that $L_{y}$, the imposed periodicity, should be of a few tens times as large as the flame thickness. However, before working with droplets, single-phase numerical experiments with strong initial noise have established that the minimal channel width allowing the DL-instability development, and denoted here by $L_{D L}$, presently corresponds to about 14 times the flame thickness (i.e. $L_{D L} \approx 14$ ). In other words, $L_{y}>L_{D L}$ is needed for observing the DLinstability in single-phase premixture, and the threshold $L_{y}=L_{D L}$ is the 
so-called cut-off scale of DL instability. Next, we consider two situations of DNS: a transversally small box (i.e. $L_{y}<L_{D L}$ ) and a transversally large box (i.e. $L_{y}>L_{D L}$ ). richer, the velocity of which finally vanishes. This rich flame accompanies 


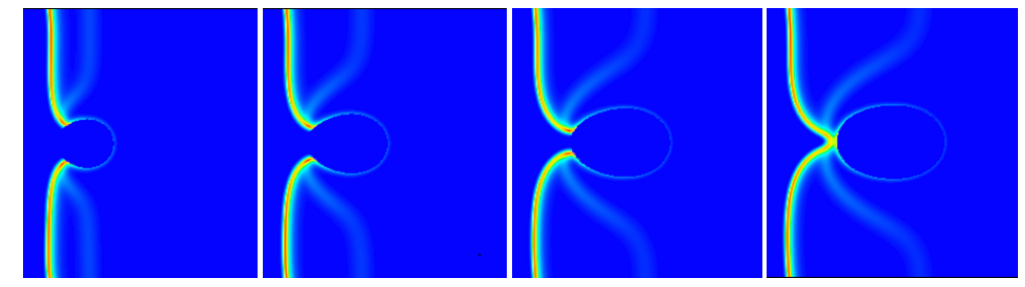

Figure 2: Four successive snapshots of the reaction rate, when a planar spray-flame interacts with a droplet in the lean case of initial surrounding mixture $\left(\varphi_{G}=0.9, \varphi_{L}=0.5, s=24, P e_{s}>\mathcal{O}(1)\right)$.
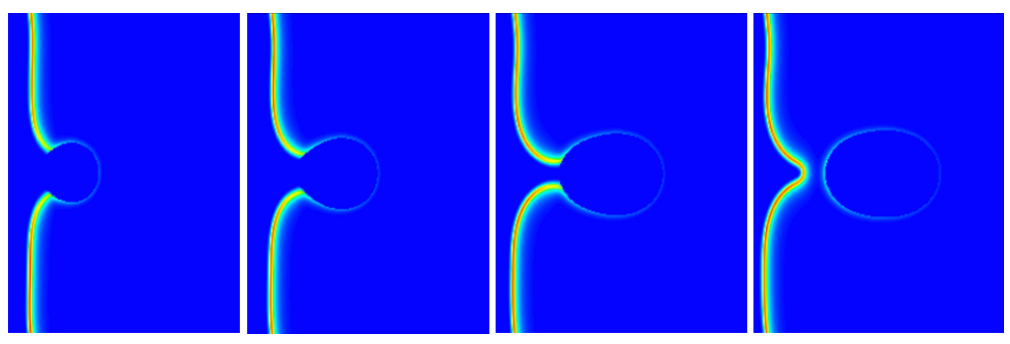

Figure 3: Four successive snapshots of the reaction rate, when a planar spray-flame interacts with a droplet in the rich case of initial surrounding mixture $\left(\varphi_{G}=1.1, \varphi_{L}=0.5, s=24, P e_{s}>\mathcal{O}(1)\right)$ 


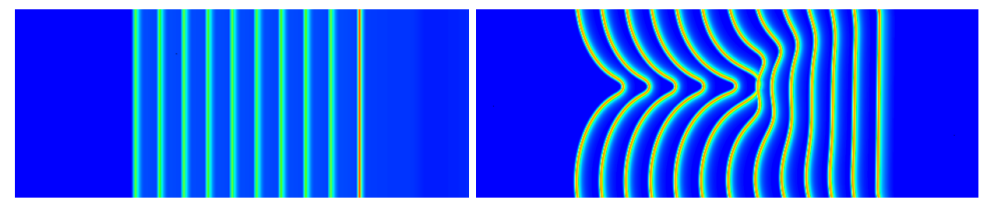

(a)

(b)

Figure 4: Superimposed successive snapshots of the reaction rate for the single-phase premixed flame $\left(L_{y}=24, \varphi_{G}=1.1\right)$; (a) stable with respect to DL-instability, since no perturbations have been applied; (b) subjected to DL-instability that has been triggered by a $y$-sinusoidal $x$-displacement of 0.3 on the initial front

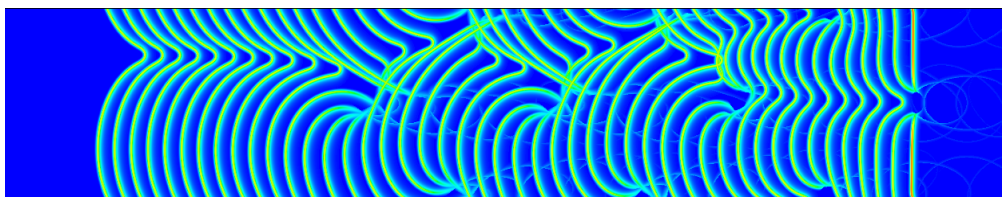

Figure 5: Superimposed successive snapshots of the reaction rate for the spray-flame subjected to DL-instability that has been triggered by droplets $\left(\varphi_{G}=1.1, \varphi_{L}=0.5, s=24\right.$ and $\left.L_{y}=24\right)$.

the droplet during its motion and takes the form of an ellipse, when carried downwards by the flow.

As for the diffusion flame, it delimits the remaining oxygen located behind the lean wing. Since the overall equivalence ratio is rich, the diffusion flame burns the rest of oxygen. The figure 2, which presents a sequence of four snapshot of the reaction rate, illustrates how the lean flame is locally deformed by the presence of a droplet.

On the other side, when the initial surrounding premixture is already rich, no triple flame exists and the presence of the droplet only modifies the equivalence ratio of the mixture in its surroundings. In other words, the flame that essentially burns the initial premixture meets rich puffs of mixture at the nodes of the lattice, which locally hinders the spray-flame propagation. In the forthcoming section, it will nevertheless be shown that those loci of hindrance induce folds that increase the overall propagation of the spray-flame. A sequence of four snapshots for the present rich case is provided in Fig. 3. 


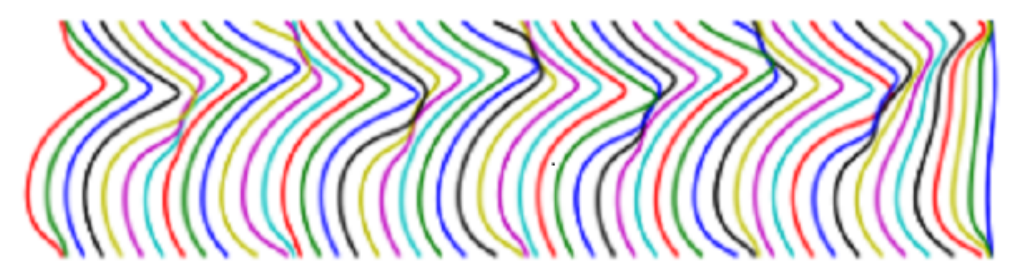

Figure 6: Successive front positions provided by the Sivashinsky DL-model equation triggered by "combustion holes"

Next, we describe how our 2D numerical experiments by DNS clearly show that the presence of droplets triggers the Darrieus-Landau instability. More precisely, we actually focus on three different situations:

- the first one considers a single-phase flame of equivalence ratio 1.1

325 (i.e. $\varphi_{G}=1.1$ and $\varphi_{L}=0$ ). A planar flame is initiated without initial perturbation on the right hand side of a $24 \delta_{f}^{*}$ high computational box. In absence of noise or perturbations, the flame remains flat, as shown in Fig. 4 a, where we have superimposed 10 successive snapshots of the reaction rate field.

330 - the second situation concerns the same single-phase flame, still initiated on the right hand side of a $24 \delta_{f}^{*}$ high box, but the front has been perturbed by a $y$-sinusoidal $x$-displacement of 0.3 times the flame thickness. As long known [21], this perturbation is sufficient to trigger the DL instability on a relatively short distance as reported in Fig. 4.b, where are superimposed 13 successive snapshots of the reaction rate.

- the third case treats of a planar spray-flame with the same equivalence ratio of the initial surrounding mixture. But this initially unperturbed flat flame meets droplets, the perturbations of which initiate the DL instability of the spray-flame. The parameters of this numerical experiment are $\varphi_{G}=1.1$, $\varphi_{L}=0.5, s=24$ and $L_{y}=24$. As indicated in Fig. 5, the presence of droplets triggers the folding of the spray-flame which then experiences rather complex corrugations. One the left-hand side of Fig. 55 (i.e. when leaving the lattice), a single-phase premixture is retrieved and we can recognize the typical non-linear DL-shape of the single-phase premixed flame.

\section{$345 \quad 3.3$ Sivashinsky DL-model equation (SME) results}

As described above, the SME approach mimics the presence of droplets thanks to holes of partial extinction distributed at the nodes of the lattice. 
The box height is here fixed to $L_{y}=s$. The simulations of the Sivashinsky equation are carried out with the following parameters; density ratio between burnt and unburnt gases equals to 5 ; number of unstable Darrieus Landau modes: 2.4; the effective flame velocity inside the droplet-affected zone is fixed to 0.2 times the laminar flame velocity; the droplet-affected zone is inside a radius of influence around the droplets, which equals 0.1 times the total width $L_{y}$. These values have been chosen in agreement with the 2D simulations of the flame propagating in the (rich) close vicinity of the droplets.

The front shape at different times is plotted in Fig. 6, showing the effect of the droplets on the hydrodynamic instability. When the front reaches a droplet, the associated partial quenching creates wrinkles which triggers the DL instability, and then sustains the particular pattern of a DL affected front.

\section{Droplets enhance spreading of DL-unstable spray- flames}

The purpose of the present section concerns the assessment of the velocity of 365 the spray-flames that are subjected to Darrieus-Landau instability, or equivalently what occurs when a single-phase flame subjected to DL instability meets a lattice of droplets.

\subsection{SME approach}

We first present the results obtained with the Sivashinsky DL-model equation. The simulations are with and without droplets, for the following numbers of unstable Darrieus-Landau modes: 1.2, 2.4 and 4.8 (1.2 corresponds to a small width). We here use initial conditions allowing the flame to reach quickly a shape with one large cell in the domain, and we now show the effect of the number of unstable modes (or equivalently domain height $L_{y}$ ) on the observed propagation velocity. The mean position of the front versus time is plotted in Fig. 7. It can be noticed in the figure that for a small width $(1.2$ unstable modes) there is not much difference between the results without droplets (black, dashed line) and with droplets (black, solid line). With 2.4 unstable modes (magenta -or dark grey-, dashed/dotted without droplets, magenta - or dark grey-, solid line with droplets), the perturbations caused by the droplets lead to larger flame amplitude and flame speed that in the case without droplets. This effect is even more important in the case of 4.8 


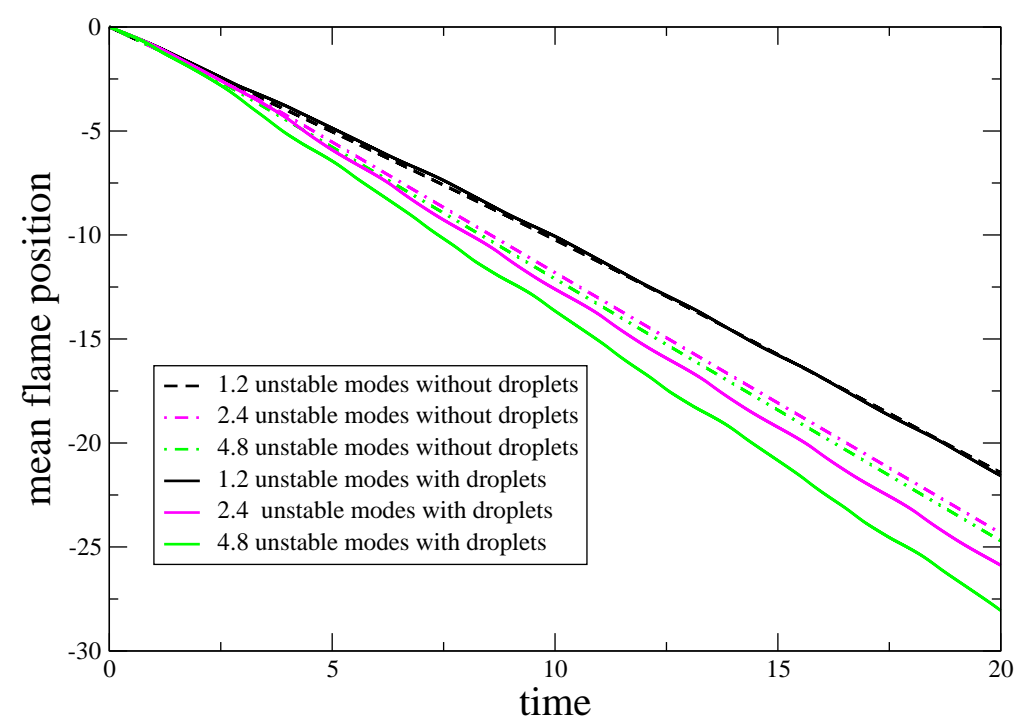

Figure 7: Mean front position vs. time computed with the Sivashinsky equation: when the number of unstable DL modes increases, the slope enhancement due to droplets is more marked.

unstable modes, green -or light grey-, dashed/dotted line without droplets, green -or light grey-, solid line with droplets.

Evidently, in what follows, the DNS approach provides us with more details on the manner that allows a flame to propagate through a lattice of droplets. However, the SME approach, implies much lighter computational efforts and allowed us to investigate a larger field of parameters. It also serves to highlight the most important (and quite paradoxical) point of this paper: the flame speed of a DL-unstable flame is increased because the flame propagates more slowly around the droplets. We will see below that the same results are obtained in our DNS.

\subsection{DNS approach}

We carry out the following DNS numerical experiments. Before meeting droplets, a single-phase flame (characterized by the set of parameters $\varphi_{G}$, $\left.\varphi_{L}=0, L_{y}\right)$ exhibits folds because it develops the DL instability from an initial perturbation, and propagates towards the lattice of droplets. The initial conditions are such that the DL instability reaches its non-linear regime before the single-phase flame meets the first droplet. On the right hand side of Fig. 8, the single-phase flame folds as it propagates towards the droplet. 


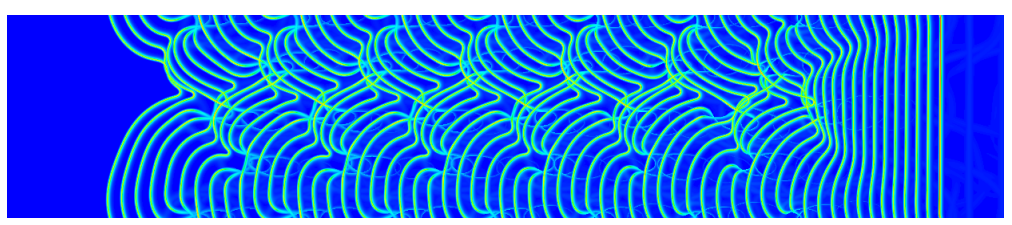

Figure 8: Superimposed successive snapshots of the reaction rate for the spray-flame subjected to subjected to Darrieus-Landau instability (DL) that has been initially triggered outside the droplets $\left(\varphi_{G}=0.9, \varphi_{L}=0.5, s=24\right.$ and $y$-periodicity of $L_{y}=48$ ).

When the first droplet is on the point to be met, the flame adopts the classical cusped shape of the DL instability. Then, when the flame propagates within the droplet lattice (i.e. we have now $\varphi_{L}=0.5$ ), it is however noticeable that the front pattern is more corrugated than in the single-phase DL instability. On the left hand side of the figure, the flame finally leaves the droplet lattice and tends to recover the classically cusped pattern.

We have performed a series of such numerical experiments for various $\varphi_{G}$ and $s$, the liquid loading of the droplet lattice being set to $\varphi_{L}=0.5$ and $L_{y}=s$ or $L_{y}=2 s$. In Fig, 9 , where combustion front x-position is plotted against time, we observe that the slope of the curve is not constant. It increases as the flame propagates within the lattice. It is now clear that the presence of droplets enhances the propagation of the DL-affected flame. In the figure, it is also observed that the change in slope occurs whatever $\varphi_{G}$, the most intense effect corresponding to the nearly stoichiometric conditions of the surrounding mixture (i.e. $\varphi_{G} \approx 1.1$ ).

The slope of the combustion $x$-positioning vs. time within the lattice is hereinafter called spray-flame speed. The magnitude of this quantity incorporates two cooperative effects: the velocity increase due to the DarrieusLandau instability which cooperates with the additional wrinkling due to 420 the vicinity of the droplets where combustion is reduced (since too rich). Evidently, the spray-flame speed will depend on the height of the computational domain $\left(L_{y}\right)$, since DL single-phase flame speed depends on $L_{y}$. Furthermore, the spray-flame speed also depends on the droplet size. But, it turns out that this effect remains weak, the number of the droplets (i.e. the role of $s$ ) for given $L_{y}$ and $\varphi_{L}$ playing a more important role.

To illustrate the essential part of these results, we have gathered the spray-flame speeds as a function of $\varphi_{T}$ for various values of $s$ and $L_{y}$, and for fixed $\varphi_{L}=0.5$. This corresponds to Fig. 10, which requires several comments. First of all, the curves with label $s=0$ corresponds to a situ- 


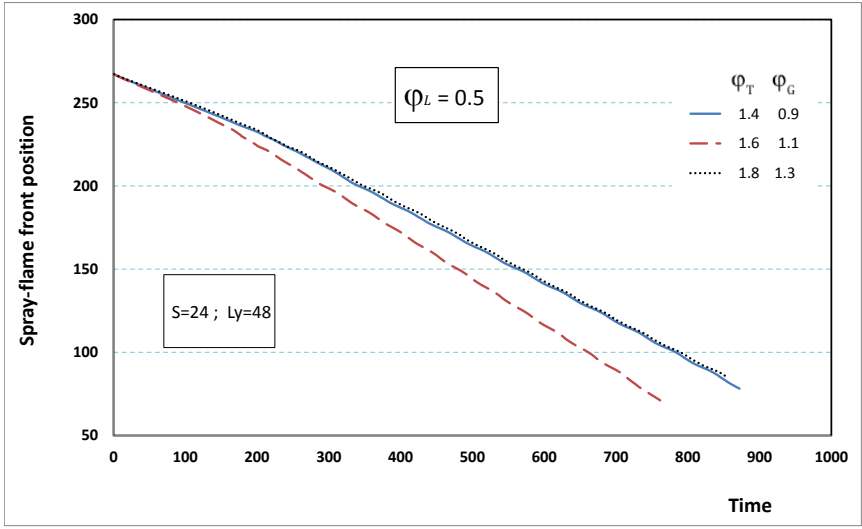

Figure 9: x-position of the combustion front vs. time for three different initial surrounding mixtures $\varphi_{G}=0.9, \varphi_{G}=1.1$ and $\varphi_{G}=1.3$; DL-affected flame accelerates when entering the droplet lattice $\left(\varphi_{L}=0.5\right.$ and $L_{y}=2 s=$ $48)$.

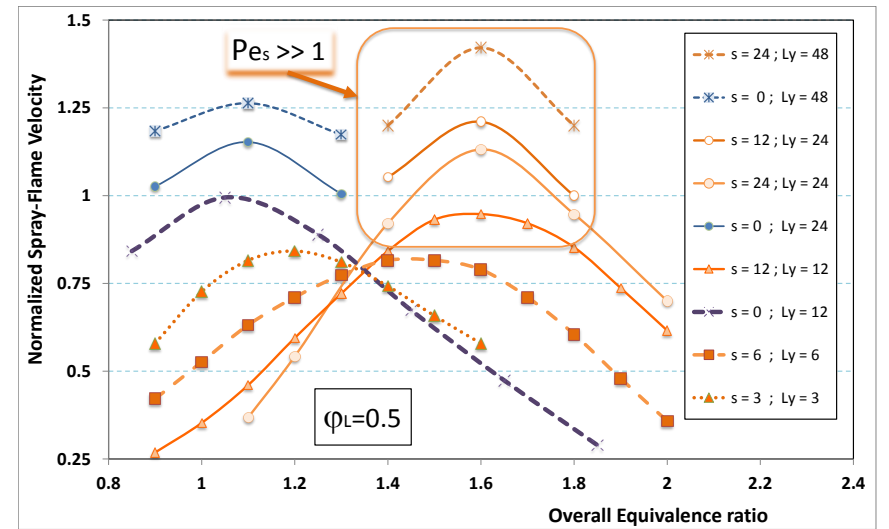

Figure 10: Normalized spray-flame speed as a function of overall equivalence ratio for various $s$ and $L_{y}$, the liquid loading being fixed to $\varphi_{L}=0.5$ (curves labelled $s=0$ correspond to the single-phase flames). 


\section{Discussion and conclusion}

The numerical experiments of the paper actually concern the propagation of three successive different flames: (a) a single-phase premixed flame of equivalence ratio $\varphi_{G}$ that meets droplets and becomes unstable versus DarrieusLandau instability; (b) a spray-flame -in the $P e_{s}>\mathcal{O}(1)$ regime- fully subjected to DL instability, for which the droplets create additional wrinkles; (c) a single-phase premixed flame of equivalence ratio $\varphi_{G}$ that leaves the domain occupied by droplets and remains unstable versus Darrieus-Landau instability.

To sustain the discussion and illustrate the point, in Fig 11 we have simultaneously drawn two pictures, namely, a picture that show the various successive front patterns, using numerous snapshots of the reaction rate field, and a graph that depicts the mean front position, the slope of the latter curve giving the front velocity. Hence, the mean velocity that corre- 


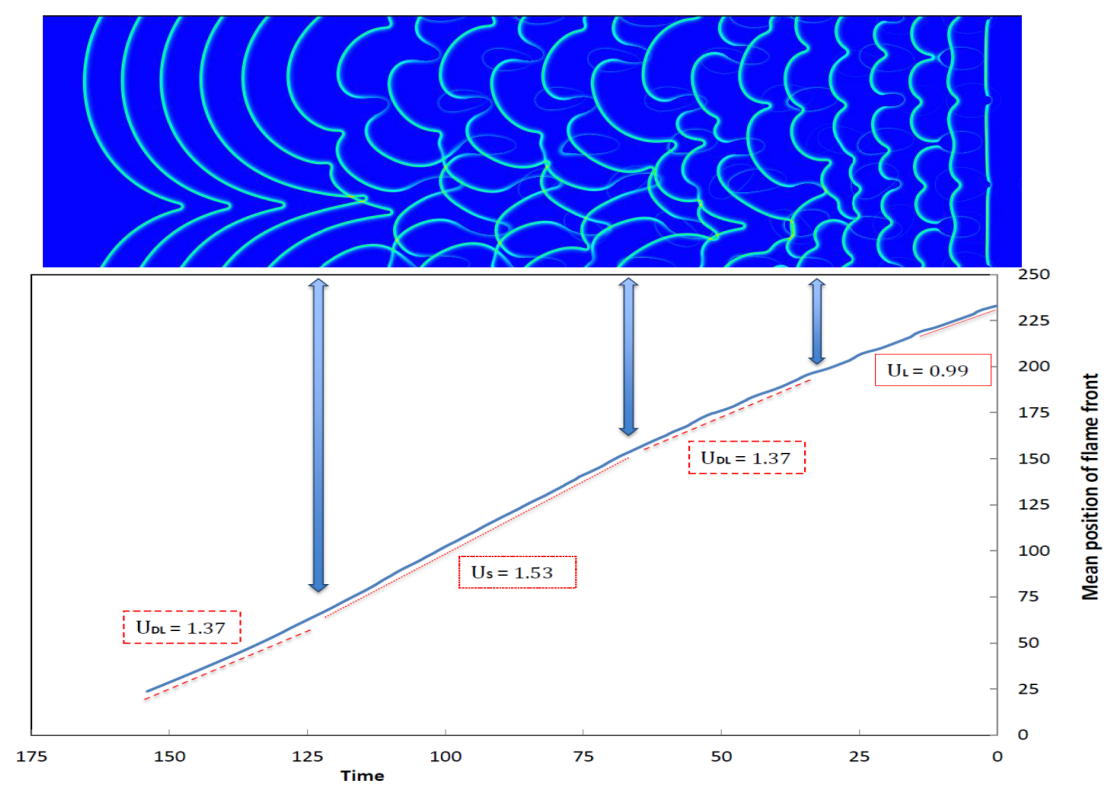

Figure 11: Superimposition of twenty one snapshots of the reaction rate field, positioned above the plot of the mean flame location, in such a way that the instant of each snapshot can be read on the time abscissa of the mean front location curve; four zones of particular flame speed are pointed out; parameters are $L_{y}=3 s=72, \varphi_{G}=1.1$ and $\varphi_{L}=0.5$ 
sponds to a particular flame pattern can be read on the position curve, as the slope of the curve vertically below the considered pattern. Fig 11 invites us to identify four different periods when the front propagates: from the 470 right to the left of the figure, we notice

- a first time during which the flame speed remains constant at about $U_{L}\left(\varphi_{G}\right)$, the quasi-planar front pattern being perturbed by the droplets, acting as "holes of weak combustion".

- a second period where the previous perturbations trigger an instability of

475 much larger amplitude and length scale, namely the Darrieus-Landau instability, which superimposes on the droplet-induced pattern. It corresponds to a period of flame speed-up.

- a third time during which the flame propagation attains a quasi-steady state of high velocity, i.e. $U_{s}=1.53$. The front pattern corresponds to the addition of two kinds of wrinkles, namely, the DL corrugations which are now of maximum amplitude, complemented with droplet-induced foldings which appear as perturbations of length-scale smaller than the DL corrugations.

- the final period corresponds to the propagation of a single-phase flame subjected to DL-instability. The corresponding (last) part of the position curve indicates a constant slope at about $U_{D L}=1.37$, which retrieves the propagation velocity of a DL-unstable premixed flame in a periodic box of 72 times the flame thickness.

The above sequence of events confirms that droplets trigger the DL-instability, then enhance propagation velocity of the DL-unstable front. In other words, DL-unstable spray-flame is faster than DL-unstable single-phase flame.

To sum-up, the Darrieus-Landau instability has been studied in the framework of spray combustion, where we have focused on the simplest sit495 uation of spray-flames that corresponds to $P e_{s}>\mathcal{O}(1)$, i.e. at large "spray Péclet number" the planar spray-flame propagates at the same rate as the single-phase premixed flame that shares the same initial fuel-saturated mixture as the spray. Two numerical approaches -a DNS and a Sivashinsky model equation- have brought results in profound agreement. In particular, 500 it has been shown that the droplet action on the premixed flame triggers the Darrieus-Landau instability on a reasonably short distance of propagation. This result is in agreement with the classical results on DL instability, since DL instability is known to require perturbations or noise to develop in a satisfactory manner [21]. The same triggering effect has been 505 obtained when including "holes of slow combustion" in SME. Furthermore, the DL-cells that appear in the experiments by Chauveau et al. [2] or in the 
Bradley-Lawes experiments [1] are of sub-centrimetric length scales. Now, one centimetre corresponds in the experiments to about 25 times the flame thickness. Therefore, our computational domain with $L_{y}=48$ can simulate

510 several practical DL-cells, which is enough to numerical handle the overall DL-dynamics.

A more striking result established in the paper, either by DNS or by SME, concerns the role of the droplets on the flame speed in the context 515 of the fully-developed Darrieus-Landau instability. The DL instability in the nonlinearly saturated regime appears as a folded front with only one cusp, which corresponds to the DL non-linear pattern with the fastest propagation. Such a front perturbed by "holes of slow combustion" exhibits additional wrinkles of shorter wavelengths. Interpreted accordingly with a linear point of view in DL-theory, those short wavelengths would lead the spray-flame to a slower propagation. By contrast, a spread enhancement is observed. This spray-flame speed promotion has to be interpreted in a non-linear manner, in the sense that secondary wrinkles are additionally imposed to the fastest DL-pattern, and still increase the effective flame surface. Such a phenomenon of secondary folds often results from a small scale turbulence, that transports the flame in both positive and negative manners with respect to propagation. Here, the droplets always act in a negative manner, the droplets locally slowing down the propagation. Nevertheless, local slowdown allows the spray-flame to accelerate. Furthermore, both approaches (DNS and SME) agree with each other, claiming that an important mechanism related to the rich spray-flame speed enhancement at high "spray Péclet number" is due to the simple fact that a droplet produces a (rich) zone of weak combustion, which induces additional folds; we are hence faced with the quite paradoxical situation that local quasi-extinction 535 can accelerate overall propagation.

\section{Acknowledgements}

The present work has received the support of the Research Program "Micropesanteur Fondamentale et Appliquée", GDR CNRS n2799, under contract CNES/150515. 


\section{${ }_{540}$ References}

[1] D. Bradley, M. Lawes, S.Y. Liao, and A Saat. Laminar mass burning and entrainment velocities and flame instabilities of isooctane, ethanol and hydrous ethanol/air aerosols. Combust. and Flame, 161(6):1620$1632,2014$.

${ }_{545}[2]$ R. Thimothee, C. Chauveau, F. Halter, and I. Gokalp. Rich spray-flame propagating through a 2D-lattice of alkane droplets in air. Proceedings of ASME Turbo Expo 2015: Turbine Technical Conference and Exposition GT2015, 62015.

[3] S. Hayashi and S. Kumagai. Flame propagation in fuel droplet-vaporair mixtures. Proc. Combust. Inst., 15:445-451, 1975.

[4] S. Hayashi, S. Kumagai, and T. Sakai. Propagation velocity and structure of flames in droplet vapor air mixtures. Combust. Sci. and Tech., 15:169-177, 1977.

[5] H. Nomura, I. Kawasumi, Y. Ujiie, and J. Sato. Effects of pressure on flame propagation in a premixture containing fine fuel droplets. Proc. Combust. Inst., 31:2133-2140, 2007.

[6] H. Hanai, K. Maruta, H. Kobayashi, and T. Niioka. Pulsating flame propagation of pmma particle cloud in microgravity. Proc. Combust. Inst., 27:2675-2681, 1998.

560 [7] F. Atzler, F. X. Demoulin, M. Lawes, Y. Lee, and N. Marquez. Burning rates and flame oscillations in globally homogeneous two-phase mixtures (flame speed oscillations in droplet cloud flames). Combust. Sci. and Tech., 178(12):2177-2198, 2006.

[8] S. Suard, P. Haldenwang, and C. Nicoli. Different spreading regimes of spray-flames. C. R. Acad. Sci. Mécanique (Paris), 332(5-6):387-396, 2004.

[9] C. Nicoli, P. Haldenwang, and S. Suard. Analysis of pulsating sprayflames propagating in lean two-phase mixtures with unity Lewis number. Combust. Flame, 143:299-312, 2005.

570 [10] J.B. Greenberg, L.S. Kagan, and G.I. Sivashinsky. Propagation of rich premixed polydisperse spray flames. Int. Journal of Spray and Combustion Dynamics, 2(4):285-300, 2010. 
[11] H. Nomura, M. Koyama, H. Miyamoto, Y. Ujiie, J. Sato, M. Kono, and S. Yoda. Microgravity experiments of flame propagation in ethanol droplet vapor air mixture. Proc. Combust. Inst., 28:999-1005, 2000.

[12] C. Nicoli, B. Denet, and P. Haldenwang. Lean flame dynamics through a 2D lattice of alkane droplets in air. Combust. Sci. and Tech., 186(2):103-119, 2014.

[13] C. Nicoli, P. Haldenwang, and B. Denet. Numerical study of flame dynamics through a 2D-lattice of alkane droplets in air. ECM2013: 6th European combustion meeting. Lund, Sweden, 62013.

[14] C. Nicoli, B. Denet, and P. Haldenwang. Rich spray-flame propagating through a 2D-lattice of alkane droplets in air. Combustion and Flame, 162(12):4598-4611, 2015.

585 [15] C. Nicoli, B. Denet, and P. Haldenwang. Spray-flame dynamics in a rich droplet array. Flow Turbulence and Combustion, 96(2):377-389, 2016.

[16] M. Nassouri, C Chauveau, F. Halter, and I. Gokalp. Flame structure of ethanol-air premixed mixtures at high pressures in microgravity. ECM2013: 6th European combustion meeting. Lund, Sweden, 2013.

[17] V. Karlin and G. Sivashinsky. Asymptotic modelling of self-acceleration of spherical flames. Proc. Combust. Inst., 31:1023-1030, 2007.

[18] D. H. Wacks and N. Chakraborty. Flame structure and propagation in turbulent flame-droplet interaction: A direct numerical simulation analysis. Flow, Turbulence and Combustion, 96(4):1053-1081, 2016.

[19] B. Lewis and G. von Elbe. Combustion, Flames and Explosions of Gases. Academic Press, 3rd edition, 1987.

[20] C. Nicoli and P. Haldenwang. Analysis of one-step chemistry models for flame propagation in various equivalence ratio premixtures of high alkane-air. SPEIC10: Towards Sustainable Combustion, Tenerife, 6 2010 .

[21] B. Denet and P. Haldenwang. A numerical study of premixed flames Darrieus-Landau instability. Combust. Sci. and Tech., 104:143-167, 1995. 
605 [22] G. Joulin and P. Cambray. On a tentative approximate evolution equation for markedly wrinkled flames. Combust. Sci. and Tech., 81:243256, 1992.

[23] B. Denet. Stationary solutions and Neumann boundary conditions in the Sivashinsky equation. Phys. Rev. E., 74:036303, 2006. 